\title{
“Happy heart syndrome” expressed as focal takotsubo
}

\author{
Sindrome del "corazón feliz" expresado como takotsubo focal \\ Manuel Chacón-Diaz*, Manuel Horna-Noriega and Cesar Conde-Vela \\ Servicio de Cardiología, Clínica Delgado, Auna, Lima, Perú
}

\section{Introduction}

Since its first description, Takotsubo syndrome has been related to unpleasant events such as physical or emotional stress ${ }^{1,2}$. In recent years, it has also been related to "positive" emotions giving way to the term "happy heart syndrome"3. In addition to the typical location of this syndrome (apical ballooning), there have been reports of atypical cases that include midventricular, basal, and focal locations ${ }^{4}$.

\section{Case report}

A 61-year-old woman with a history of arterial hypertension, hyperglycemia, hypothyroidism, and hypercholesterolemia complains of oppressive precordial pain (not irradiated) after knowing that she would be grandmother. This discomfort lasted about $20 \mathrm{~min}$ and disappeared after habitual antihypertensive intake. After $4 \mathrm{~h}$, she again presents the same symptom at rest, going to emergency.

At admission, no angina was present. She had stable vital functions, cardiovascular and respiratory examination was normal. The electrocardiogram of admission showed negative $T$ waves in leads V1 to V3 (Fig. 1A). Admission analysis showed ultrasensitive troponin $T$ in $0.232 \mathrm{ng} / \mathrm{ml}$ (normal value: $0.0-0.05 \mathrm{ng} / \mathrm{ml}$ ), low-density lipoprotein cholesterol $168 \mathrm{mg} / \mathrm{dl}$, high-density lipoprotein cholesterol $47 \mathrm{mg} / \mathrm{dl}$, glucose $106 \mathrm{mg} / \mathrm{dl}$, and hemoglobin $13.3 \mathrm{~g} / \mathrm{dl}$.

With the above data and the diagnosis of non-ST elevation myocardial infarction, we initiated anti-ischemic therapy with clopidogrel $600 \mathrm{mg}$ orally, intravenous nitrates, beta-blocker, and subcutaneous anticoagulation. Due to the antecedent of aspirin allergy, desensitization was carried out with progressive doses in a period of $2 \mathrm{~h}$. The echocardiographic study reported the left ventricular ejection fraction of $52 \%$, apical and midanteroseptal hypokinesia, and left ventricular filling pattern Type I (Fig. 1B). With approximately $12 \mathrm{~h}$ of symptoms onset, coronary angiography was performed (Fig. 2), no coronary lesions were found, ventriculography showed localized akinesia in the anterior wall.

The evolution was favorable without clinical signs of heart failure or new episodes of angina. She was discharged after 4 days with no symptoms, taking candesartan, statins, and aspirin. One week later, the echocardiographic follow-up showed total resolution of the motility alterations (Fig. 1C), confirming the diagnosis of focal Takotsubo syndrome. The electrocardiographic changes remained until the control 3 months later.

\section{Discussion}

Takotsubo syndrome presents as an acute coronary syndrome characterized by the absence of "potentially

\section{Correspondence:}

*Manuel Chacón-Diaz

E-mail: manuelchacon03@yahoo.es

DOI: 10.24875/ACM.M19000003
Available online: 19-03-2019 Arch Cardiol Mex. 2019;89(1):85-87 www.archivoscardiologia.com la licencia CC BY-NC-ND (http://creativecommons.org/licenses/by-nc-nd/4.0/). 


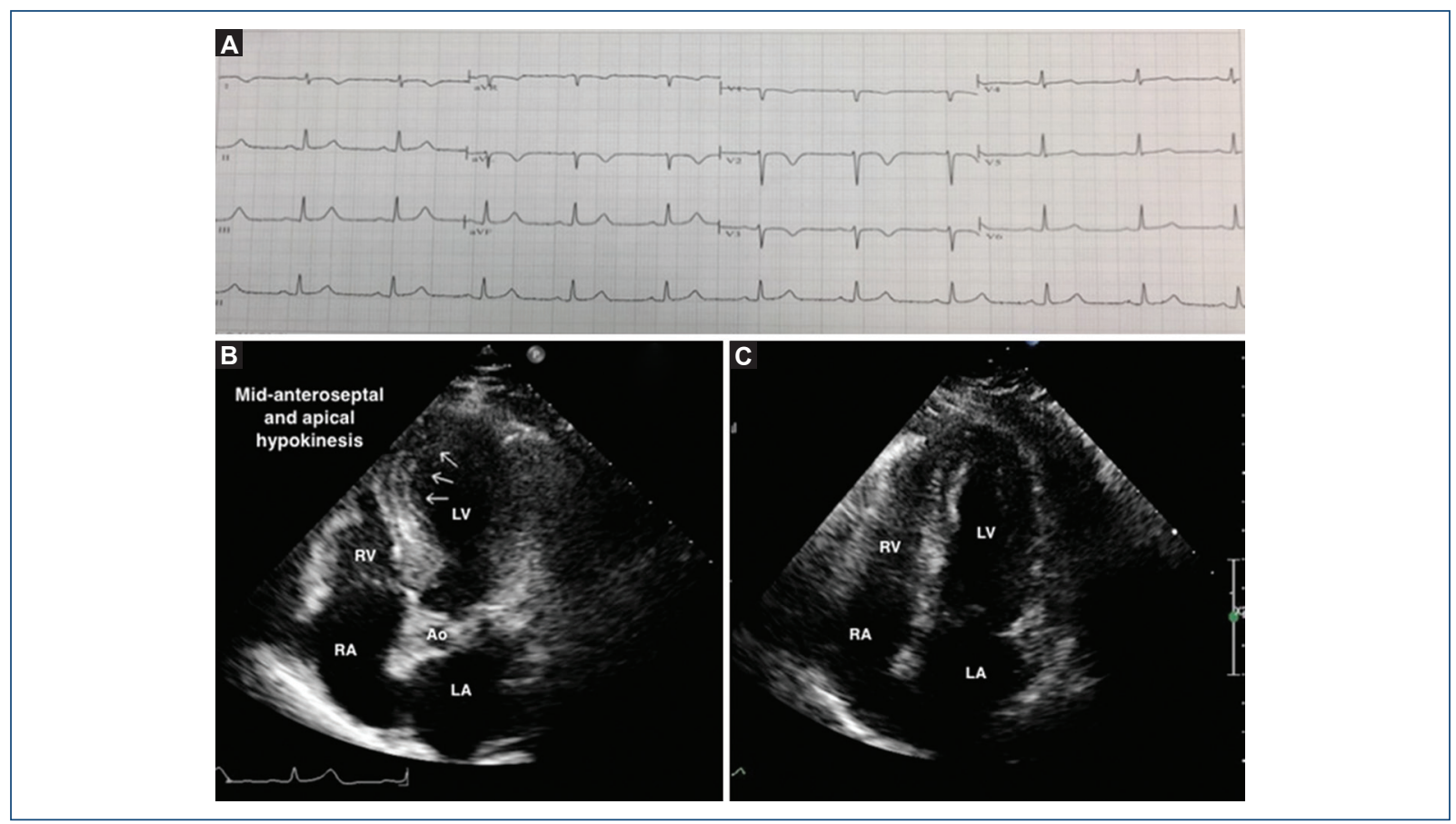

Figure 1. A: Electrocardiographic features at admission, showing negative T waves on V1 to V3, DI, and a VL leads. B: echocardiogram (apical view) on admission shows medial and apical anteroseptal wall hypokinesis (arrows) and compensatory contractility of the rest of the walls, LVEF: $52 \%$. C: 8 days later, echocardiogram (apical view) shows normalization of previously hypokinetic segments, LVEF: $62 \%$. LVEF: left ventricular ejection fraction; LA: left atrium; RA: right atrium; LV: left ventricle; RV: right ventricle; Ao: aorta.

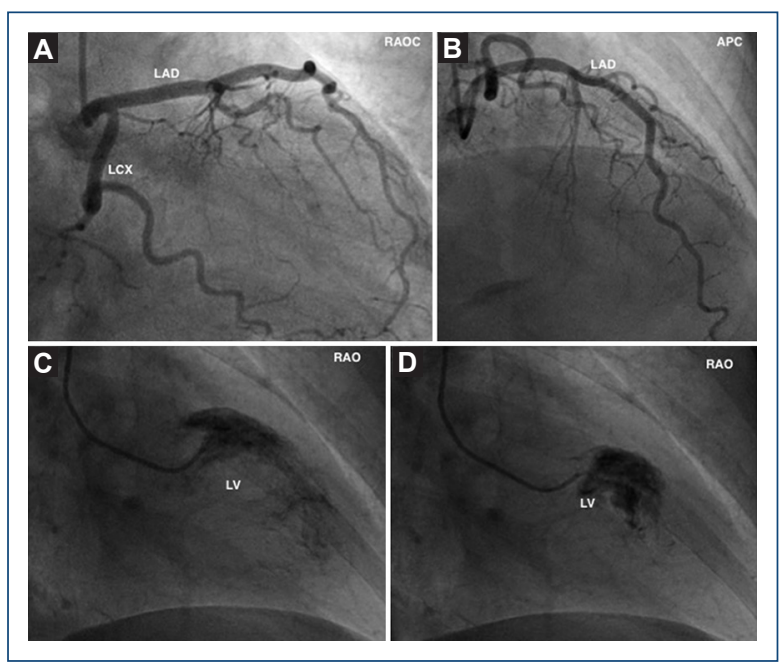

Figure 2. A and B: Coronary angiogram showing LAD and LCx arteries without obstruction. C: Left ventriculography in diastole. D: Left ventriculography in systole with contrast retention in the midanterior wall. (AD: left anterior descending; LCx: left circumflex; RAOC: right anterior oblique caudal view; APC: anteroposterior cranial view; RAO: right anterior oblique view; LV: left ventricle.

guilty" coronary obstructions with or without ventricular dysfunction that typically recovers in days or weeks and is usually preceded by emotional or psychological stress ${ }^{5}$.
Clinical presentation can range from chest pain and dyspnea to cardiogenic shock or serious ventricular arrhythmias. In the electrocardiogram, it usually presents as elevation of the ST segment in precordial leads without reciprocal changes ${ }^{6}$. In some cases, T-wave inversion is present as in the case of our patient. The elevation of troponin is usually slight compared to the ST elevation myocardial infarction ${ }^{5}$. Up to $15 \%$ of patients have coronary obstructions, in which case Takotsubo is suspected due to the extension of cardiac dysfunction comprising more than one coronary territory and its reversibility ${ }^{4}$.

Recently, the International Registry of Takotsubo (InterTAK registry) has reported that the focal presentation of the syndrome is around $1.5 \%{ }^{4}$.

Many studies have linked an increase in catecholamines with myocardial damage directly and indirectly, and spontaneous resolution seems to be related to the activation of survival pathways such as those seen in post-ischemic myocardial stunning ${ }^{5}$.

According to the InterTAK registry ${ }^{3}$, the "happy heart syndrome" - a type of Takotsubo syndrome caused by "positive" emotions - represents just $1.1 \%$ of the study population ( $4 \%$ of the cases produced by emotional triggers), and, in contrast to the one produced by "negative" emotions, it presents more frequently with ST-segment 
depression (15\% vs. $5 \%$ ) and with the midventricular type (35\% vs. $16.3 \%$ ). In this study, they also linked all the focal-type cases to negative emotions, so our case is - to our knowledge - the first reported case of focal Takotsubo produced by a "happy heart syndrome."

\section{Funding}

None.

\section{Conflicts of interest}

The authors declare no conflicts of interest.

\section{Ethical disclosures}

Protection of human and animal subjects. The authors declare that no experiments were performed on humans or animals for this study.
Confidentiality of data. The authors declare that they have followed the protocols of their work center on the publication of patient data.

Right to privacy and informed consent. The authors declare that no patient data appear in this article.

\section{References}

1. Akashi YJ, Goldstein DS, Barbaro G, et al. Takotsubo cardiomyopathy: a new form of acute, reversible heart failure. Circulation. 2008;118:2754-62.

2. Sharkey SW, Lesser JR, Zenovich AG, et al. Acute and reversible cardiomyopathy provoked by stress in women from the United States. Circulation. 2005;111:472-9.

3. Ghadri J, Sarcon A, Diekmann J, et al. Happy heart síndrome: role of positive emotional stress in takotsubo síndrome. Eur Heart J. 2016;37:2823-9.

4. Templin C, Ghadri J, Diekmann J, et al. Clinical features and outcomes of takotsubo (stress) cardiomyopathy. N Engl J Med. 2015;373:929-38.

5. Pellicia F, Kaski J, Crea F, et al. Pathophysiology of takotsubo syndrome. Circulation. 2017;135:2426-41.

6. Kurisu S, Inoue I, Kawagoe T, et al. Time course of electrocardiographic changes in patients with tako-tsubo syndrome: comparison with acute myocardial infarction with minimal enzymatic release. Circ J. 2004;68:77-81. 